\title{
TAGUNG
}

\section{Die innere und äußere Sicherheit der Europäischen Union - eine Standortbestimmung}

\author{
Tobias Heller*
}

Schutz einer Gesellschaft und eines Staatenbundes bedeutet, den Bürgern innere und äuBere Sicherheit gewähren zu können. Dass ein erhöhter Handlungsbedarf der Europäischen Union als sicherheitspolitischer Akteur besteht, hat sich nicht zuletzt eindringlich am Beispiel des Kaukasuskonflikts und dem damit verbundenen Umgang mit Russland gezeigt sowie an dem immer noch bedrohlichen internationalen Terrorismus, der stetig zunehmenden Intensität illegaler Migration in den EU-Raum und den anhaltenden Prozessen der Globalisierung. Angesichts dieser Herausforderungen stellt sich die Frage nach der Handlungsfähigkeit der Europäischen Union, ihren Kompetenzen, Instrumenten und ihrer demokratischen Legitimität. In Anbetracht eines zunehmenden Ineinandergreifens von Fragen innerer und äußerer Sicherheit offenbart sich das Feld als eines der dynamischsten in der Europäischen Union und von langfristiger Bedeutung. Diese Verschränkungen und der enge Zusammenhang von innerer und äußerer Sicherheit seien jedoch von der Forschung noch nicht ausreichend erschlossen worden und verdienten eine Standortbestimmung, betonte der Vorsitzende des Wissenschaftlichen Direktoriums Michael Kreile in seiner Einführung in die Thematik der interdisziplinären Konferenz des Instituts für Europäische Politik (IEP).

$\mathrm{Zu}$ den Referentinnen und Referenten zählten Vertreter der Bundesregierung, Mitglieder des Wissenschaftlichen Direktoriums des IEP sowie weitere außen- und sicherheitspolitische Experten (siehe Programmübersicht).

\section{Die innere und äußere Sicherheit der Europäischen Union}

Fachkonferenz des Instituts für Europäische Politik (IEP) in Zusammenarbeit mit dem Wissenschaftlichen Direktorium mit Unterstützung des Auswärtigen Amtes, der Europäischen Kommission sowie der Fritz Thyssen Stiftung

Berlin, 18./19. September 2008

\section{Begrüßung}

Dietlind JERING, Kommissarische Leiterin, Vertretung der Europäischen Kommission in Deutschland, Berlin

Prof. Dr. Michael KREILE, Vorsitzender des

Wissenschaftlichen Direktoriums des IEP; Humboldt-Universität zu Berlin

Prof. Dr. Mathias JOPP, Direktor, Institut für Europäische Politik (IEP), Berlin

Europapolitische Aussprache: die EU nach dem irischen Referendum - handlungsfähig ohne Vertrag?

Elmar BROK, MdEP, Vertreter des Europäischen Parlaments bei der Regierungskonferenz 2007.

Bielefeld/Brüssel

Peter TEMPEL, Leiter der Europaabteilung,

Auswärtiges Amt, Berlin

Prof. Dr. Wolfgang WESSELS, Universität zu Köln

Einwanderung als Sicherheits-, Arbeitsmarktund Integrationsproblem

Vorsitz

Prof. Dr. Markus JACHTENFUCHS, Hertie

School of Governance, Berlin

Die gemeinschaftsrechtliche Basis der Einwanderungs- und Asylpolitik

Dr. Friedemann KAINER, Ruprecht-Karls-Universität Heidelberg

Immigration und Sicherheit - das Projekt der französischen Ratspräsidentschaft Prof. Dr. Gisela MÜLLER-BRANDECK-BOCQUET, Universität Würzburg

\footnotetext{
* Tobias Heller, Doktorand, Institut für Europäische Politik, Berlin.
} 
Die EU bleibt handlungsfähig auch nach dem irischen Referendum

Den Einstieg in die Tagung bildete die Erörterung des Ausgangs des irischen Referendums. Trotz unterschiedlicher Akzente herrschte weitgehende Übereinstimmung, dass die Europäische Union auch nach dem gescheiterten Referendum in Irland handlungsfähig bleibe und folglich Entscheidungen auf der Basis der bestehenden Verträge (Nizza) durchsetzbar seien. Der Grund für das Scheitern des Referendums wurde unter anderem der Organisation „Libertas“ zugeschrieben, die die Wähler gezielt gegen den Lissabonner Vertrag mobilisierte. Umfragen zufolge fühlte sich außerdem die irische Bevölkerung nicht genügend informiert, was sich insbesondere bei Jugendlichen und sozial Benachteiligten in einer negativen Haltung bei der Abstimmung zum Lissabonner Vertrag äußerte. Unterschiedlich wurde der zukünftige Umgang mit Irland beurteilt. Während einige eine Bringschuld der irischen Regierung gegenüber der übrigen Gemeinschaft konstatierten und die Ratifizierung in den übrigen Mitgliedstaaten als externer Druck auf Irland und politisches Momentum verstanden wurde, bewerteten andere eben diese Erwartungshaltung als kontraproduktiv. Wenn der Lissabonner Vertrag zu hoch gehängt werde, bestehe im Falle eines Scheiterns die Gefahr der, self-fulfilling prophecy'. Die Folgen eines zweiten, negativen Referendums wären dann tiefgreifend. Dennoch herrschte Einigkeit darin, dass der Ratifizierungsprozess in allen übrigen Mitgliedstaaten fortgesetzt werden solle. Der Ausgang des Referendums könne nicht ausschließlich als ein irisches Problem betrachtet werden. Die Europäische Union sollte deshalb in $\mathrm{Zu}$ kunft noch deutlicher herausstellen, welche Ziele sie seit ihrer Gründung verfolgt und wie Nationalstaaten und Zivilgesellschaften von der Gemeinschaft profitieren. Den kleineren Mitgliedstaaten könne in dieser inhaltlichen Übermittlung eine besondere Verantwortung zukommen. Eine bloße Informationsoffensive erscheint hingegen zu kurz gegriffen. Der Lissabonner Vertrag beruht auf einem achtjähri-
Einwanderung, Integration und Arbeitsmarkt Prof. Dr. Richard MÜNCH, Universität Bamberg Prof. Dr. András INOTAI, Generaldirektor, Institut für Weltwirtschaft, Ungarische Akademie der Wissenschaften, Budapest

\section{Aktuelle Themen der Außenpolitik}

Dr. Christoph HEUSGEN, Leiter der Abteilung 2, Außen-, Sicherheits- und Entwicklungspolitik, Bundeskanzleramt, Berlin

\section{Wo steht die Europäisierung der inneren} Sicherheit?

Vorsitz

Prof. Dr. Franz MAYER, Universität Bielefeld

Institutionalisierte Kooperation und Umsetzungshürden in der europäischen Innenpolitik Matthias OEL, EU-Direktor, Bundesministerium des Innern, Berlin

Dr. Andreas MAURER, Stiftung Wissenschaft und Politik, Berlin

Die EU-Politik zur Terrorismusbekämpfungeine Bewertung

Raphael BOSSONG, London School of Economics (LSE); Global Public Policy Institute (GPPI), Berlin

Zehn Jahre nach St. Malo: eine Zwischenbilanz der ESVP

Vorsitz

Prof. Dr. Hartmut MARHOLD, Generaldirektor, Centre International de Formation Européenne, Nizza/Berlin

Institutionen, Ressourcen und ,strategic culture"

Dr. Heinrich KREFT, Außenpolitischer Berater der CDU/CSU-Fraktion im Deutschen Bundestag, Berlin

PD Dr. Markus KAIM, Stiftung Wissenschaft und Politik, Berlin

Krisenregionen an der Peripherie und die Leistungsfähigkeit der EU als Ordnungsmacht Prof. Dr. Hanspeter NEUHOLD, Universität Wien

Vor einer neuen sicherheitspolitischen Arbeitsteilung zwischen NATO und EU?

Prof. Dr. Walther STÜTZLE, Staatssekretär a.D.; Universität Potsdam und Stiftung Wissenschaft und Politik, Berlin

Prof. Dr. Mathias JOPP

Schlussfolgerungen

Prof. Dr. Michael KREILE

gen Prozess und eine Neuverhandlung wurde von allen Referenten ausgeschlossen. In Anbetracht der Europäischen Parlamentswahlen 2009 stellt sich die Frage, auf welcher Grund- 
lage diese Wahlen stattfinden werden. Die Zeit drängt also, eine tragfähige Entscheidung herbeizuführen. Alle zeigten sich dennoch optimistisch, dass dies bis März 2009 möglich sei.

\section{Neue Migrationskonzepte unter der französi- schen Ratspräsidentschaft}

Formen der Migrationssteuerung, die Sicherung der Außengrenzen sowie die Integrationspolitik in der Europäischen Union standen im Mittelpunkt der ersten Diskussionsrunde zu Fragen der inneren Sicherheit in der Europäischen Union. Die Europäische Union hat große Anstrengungen in diesen Politikfeldern unternommen. Ein umfassendes europäisches Migrationkonzept werde jedoch dringend benötigt, so die einhellige Meinung der Referenten. Denn die Intensität der Migration an den Außengrenzen der Europäischen Union werde eher noch zunehmen und die bisherigen Migrationsbewegungen seien nur die Spitze des Eisberges, prognostizierte ein Referent. Insbesondere wurde der steigende Migrationsdruck aus Afrika genannt. Auch die Migration innerhalb der Europäischen Union, zum Beispiel aus Rumänien und Bulgarien, hat an Intensität gewonnen. Die Motive der Migranten seien in erster Linie wirtschaftlicher und sozialer Natur. Eine kohärente Strategie und gemeinsame Migrationssteuerung der Europäischen Union sollte zwischen temporärer und dauerhafter Migration unterscheiden.

Die Initiative „Pakt über Immigration und Asyl" der französischen Ratspräsidentschaft offeriert Steuerungsmöglichkeiten für eine gemeinsame Einwanderungs- und Asylpolitik aller EU-Staaten. Die Initiative bietet Möglichkeiten für eine bessere Administration von legalen wie auch illegalen Einwanderern sowie eine bessere Koordination der Ursprungs-, Transit- und Immigrationsländer. Die gesteuerte Einwanderung, die ,immigration choisie", dient der Nachfrage von qualifizierten Immigranten. Sie soll durch neue Ansätze wie die ,zirkuläre Migration“ und die „Mobilitätspartnerschaft“, die eine befristete
Arbeitsmigration ermöglichen, unterfüttert werden. Hinsichtlich der Bekämpfung der illegalen Einwanderung kommt der europäischen Datenbank zur Speicherung von Fingerabdrücken (EURODAC) eine wichtige Funktion zu, unterstützt wird dies durch die Erfassung von biometrischen Erkennungsmerkmalen.

Zwar wurde die zunehmende Sekuritisierung bei der Gestaltung des ,Raumes der Freiheit, der Sicherheit und des Rechts" problematisiert und vor einer Schieflage gewarnt. Inwieweit die von der französischen Ratspräsidentschaft vorgeschlagenen repressiven Maßnahmen zu Lasten der Freiheit der Bürger gehen, blieb kontrovers. Dennoch waren sich die Referenten einig, dass der Wegfall der Binnengrenzen durch eine effektive Sicherung der Außengrenzen kompensiert werden müsse. Die Europäische Agentur für die operative Zusammenarbeit an den Außengrenzen der Mitgliedstaaten der Europäischen Union (FRONTEX) wurde als Beispiel dafür angeführt, wie die Koordinierung einzelstaatlicher Aktionen zur Durchführung von Gemeinschaftsmaßnahmen im Bereich des Grenzschutzes an den Außengrenzen funktionieren kann. Aus Expertensicht folgt der „Pakt über Immigration und Asyl" aber maßgeblich dem Primat der französischen Innenpolitik und ist als Antwort des französischen Präsidenten auf das Sicherheitsbedürfnis der Franzosen zu verstehen. Massenlegalisierung von illegalen Einwanderern bleibt, nicht zuletzt angesichts des liberalen Einwanderungskurses des Nachbarlandes Spanien, verwehrt. Auch wenn der Akzent eindeutig auf Sicherheit und nicht auf Freiheit liegt, enthält der Pakt progressive Ansätze und Ideen zur Migrationssteuerung.

Eine andere Perspektive stellte die Integration der Migranten in die EU-Gesellschaften in den Mittelpunkt. Mit Blick auf das Entstehen einer Mobilitätsgesellschaft wurden die aktuellen Ergebnisse der Organisation für wirtschaftliche Zusammenarbeit und Entwicklung (OECD) und die internationalen Schulleistungsuntersuchungen (PISA) angeführt. Dis- 
kutiert wurde vor allem das schlechte Abschneiden Deutschlands im europäischen Vergleich und die Chancenungleichheit für Kinder mit Migrationshintergrund. Der Schlüssel zur Integration sei der Zweitsprachenerwerb.

Außenpolitik - unterschiedliche Perzeptionen der Geschichte

Die Georgienkrise, die Intervention Russlands und die Reaktionen der Bundesregierung und der Europäischen Union bestimmten die Diskussion über aktuelle außen- und sicherheitspolitische Fragen. Beachtung fanden die Perzeptionsunterschiede. Denn weite Teile der russischen Bevölkerung, im Einklang mit der politischen Führung, sehen die Auflösung und den Untergang der Sowjetunion als Demütigung, während im wiedervereinigten Deutschland und Europa dieser historische Moment als Befreiung und Bestätigung des westlichen Systems gefeiert wird. Es liege im gegenseitigen Interesse, gute Beziehungen zu pflegen. Während Russland auf europäische Investitionen zur Modernisierung des Landes angewiesen sei, brauche Europa die Öl- und Gasvorkommen Russlands. Der NATO-Russland-Rat sollte gerade in einer schwierigen Situation als Forum des Dialogs aufgewertet werden.

Der weitere Blick auf die Krisenherde internationaler Politik fiel dann auf Afghanistan. Das zivile Krisenmanagement in Afghanistan wurde als sehr erfolgreich bewertet und ein wichtiges Augenmerk der Bundesregierung liege weiterhin auf Pakistan. Auch auf dem Balkan habe die Europäische Union eine Erfolgsgeschichte zu verzeichnen, welche zeige, dass die Europäische Union in wichtigen Fragen geschlossen auftreten könne. Das Verhältnis zwischen der Bundesrepublik und den USA basiere auf einer gleichen Wertegrundlage und auch mit der kommenden Administration, ob unter Obama oder McCain, werde eine gute Zusammenarbeit möglich sein. Für die Beseitigung der internationalen Probleme und Konflikte sei ein gutes Verhältnis der Eu- ropäischen Union und Deutschlands zu den USA zentral.

\section{Europäisierung der inneren Sicherheit: eine Bilanz}

Die Europäisierung der inneren Sicherheit bildet eine der größten ,Baustellen“ in der Europäischen Union. Dieser junge Kompetenzbereich war erst durch den Maastrichter Vertrag im Primärrecht etabliert worden. Obschon der rechtliche Rahmen im Bereich der Justiz und des Inneren weiterentwickelt worden sei und die Verzahnung mit anderen Politikbereichen der Union voranschreite, gibt es wesentliche Implementierungsdefizite. Vom „Haager Programm zur Stärkung von Freiheit, Sicherheit und Recht in der Europäischen Union" von November 2004, welches noch bis Ende 2009 gilt und politische Ziele und Leitlinien festlegt, sind nur rund 38 Prozent umgesetzt worden. Die „Zukunftsgruppe“, eine informelle Gruppe auf Ministerebene, wurde unter deutscher Ratspräsidentschaft auf Vorschlag von Bundesminister Schäuble und dem damaligen für Innen- und Justizpolitik zuständigen Vizepräsidenten der Europäischen Kommission, Franco Frattini, im Januar 2007 eingesetzt und benennt unter anderem die Umsetzungshürden des Haager Programms. Mitglieder waren neben Frattini, beziehungsweise seit Frühjahr 2008 Vizepräsident Jacques Barrot, die Innenminister der damals amtierenden und der nachfolgenden Trio-Präsidentschaft also von Deutschland, Portugal und Slowenien sowie von Frankreich, der Tschechischen Republik und Schweden. Ferner nahm jeweils ein wechselnder Vertreter der darauffolgenden Trio-Präsidentschaft aus Spanien, Belgien und Ungarn teil. Für das Europäische Parlament nahm der jeweilige Vorsitzende des Ausschusses für bürgerliche Freiheiten, Sicherheit und Recht als Beobachter teil. Auch das Generalsekretariat des Rates war mit Beobachterstatus vertreten. Die Erkenntnisse der Zukunftsgruppe gelten als wichtiger Beitrag und Anregung für die Europäische Kommission - wegen der inhaltlichen Vorschläge, aber auch wegen der durch die Zusammenset- 
zung breiten Legitimationsbasis. Ziel der Zukunftsgruppe, die im Juli 2008 in Cannes ihre Ergebnisse vorstellte, war es, über die zukünftige Gestaltung der europäischen Innenpolitik $\mathrm{zu}$ beraten und ein Nachfolgeprogramm für das Haager Programm zu erarbeiten. Die Zukunftsgruppe hat dabei drei horizontale Herausforderungen herausgestellt; nämlich den Erhalt des ,europäischen Modells“ im Bereich der europäischen Innenpolitik durch die Herstellung eines Gleichgewichts zwischen Mobilität, Sicherheit und Privatheit, die Bewältigung der zunehmenden Verflechtung von innerer und äußerer Sicherheit sowie die Gewährleistung eines optimalen Datenflusses innerhalb der europäischen Informationsnetzwerke. Ziel ist es außerdem, den Schutz der inneren Sicherheit und äußeren Stabilität durch polizeiliche Zusammenarbeit, Terrorismusbekämpfung und Einsätze in Drittstaaten zu gewährleisten. Politiker und wissenschaftliche Beobachter stimmten darin überein, dass trotz Implementierungshürden substanzielle Fortschritte gemacht worden seien. Durch den Lissabonner Vertrag könnte jedoch eine noch größere Dynamik entstehen.

$\mathrm{Zu}$ den Hürden zählt auch die Veränderung des institutionellen Gleichgewichts in der EU27, die die Bildung von Koalitionen erschwert und mit institutionellen Konflikten konfrontiert ist. Die Zusammensetzung von kleineren Gesprächskreisen wie zum Beispiel die G6 (Frankreich, Deutschland, Spanien, Polen, Großbritannien und Italien) oder das Forum Salzburg (Österreich, Polen, Slowakei, Slowenien, Tschechien und Ungarn) unterstütze zwar die politische Meinungsbildung, verstärke allerdings das Spannungsverhältnis zwischen dem Rat der Justiz- und Innenminister und der Kommission. Deshalb haben die informellen Treffen auf Ministerebene durchaus ihre Berechtigung und gelten als innovative Entscheidungsvorbereitung. Der Prozess der informellen Entscheidungsvorbereitung wurde allerdings auch kritisch beurteilt. Auch wurde die Frage der Transparenz und Legitimation für ein solches Vorgehen gestellt und eine Umakzentuierung von Sicherheit zu Las- ten der Freiheit festgestellt. Es bedürfe einer gut funktionierenden Balance zwischen der gewünschten Freizügigkeit innerhalb Europas und einem zeitgemäßen Grenzschutzsystem, das illegale Zuwanderung, organisiertes Verbrechen und Terrorismus effizient und mit modernen Mitteln bekämpfe.

Durch eine stärkere horizontale und interdisziplinäre Verflechtung sei die traditionelle Trennung von innerer und äußerer Sicherheit in der Europäischen Union jedoch nicht mehr möglich. Es sei schwierig, Synergien zwischen der inneren und der äußeren Sicherheit herzustellen, betonte ein Referent. Dies liege zum einen an der ungewissen Zukunft des Lissabonner Vertrags, zum anderen müsse man die Konsolidierung nach der europäischen Erweiterung abwarten. Der, integrative Ansatz', die innere und äußere Sicherheit miteinander zu verzahnen, bleibe aber Prämisse und Ziel zugleich.

\section{Fehlende 'strategic culture' und mangelnde Ressourcenplanung in der ESVP}

Zum Abschluss der Konferenz wurden die Fortschritte der Europäischen Sicherheitsund Verteidigungspolitik (ESVP) sowie das Verhältnis zwischen der Europäischen Union und der NATO betrachtet. Es ergab sich folgendes Bild: Trotz einer positiven Entwicklung der EU bei der Bewältigung von Krisen und Einsätzen an der Peripherie, wie zum Beispiel im Kosovo, im Kaukasus aber auch im Tschad, sei festzuhalten, dass die ESVP unter den Bedingungen intergouvernementaler Kooperationen agiere. So wurde kontrovers diskutiert, ob die ESVP als funktionale Reaktion auf externe Sicherheitsfragen $\mathrm{zu}$ verstehen und $\mathrm{zu}$ bewerten sei oder eher als Mittel, um die europäische Integration voranzutreiben. Dennoch waren sich die Referenten einig, dass für die Verbesserung der zivilen und militärischen Ressourcenplanung ein stärkerer politischer Wille unabdingbar sei. Dabei geht es im Besonderen um die zentrale Koordination der Ressourcen, die Logistik der Einsätze sowie die Finanzierung der Missio- 
nen. Die Ablehnung des Lissabonner Vertrags wurde von den Beteiligten als Rückschlag bewertet, obgleich Fortschritte für die zentrale Koordination und Logistik auch ohne Lissabon möglich sein werden. Die bisherigen Missionen seien weder durch eine gemeinschaftliche Koordination der Ressourcen noch durch eine vorausschauende Ressourcenplanung gekennzeichnet. Vielmehr reagiere die ESVP auf externe Krisen und Anforderungen. Kritisch konstatierte ein Referent, die Verteidigungspolitik sei nur marginal entwickelt und überhaupt könne man den Begriff Europäische Sicherheits- und Verteidigungspolitik in Bezug auf die Verteidigungspolitik als Etikettenschwindel bezeichnen. Auch sei keine Kooperation zwischen Europäischer Union und NATO zu erkennen. Die Europäische Union könne aus eigener Kraft weder Lösungen anbieten noch im Rückgriff auf die NATO realisieren. Sie bedarf der Zustim- mung der USA. Sie verspreche Sicherheit, müsse sich dann aber im ,Leasingverfahren " an die Alliierten wenden, betonte ein weiterer Referent. Die Abhängigkeit von der NATO sei groß, der politische Mut dieses Verhältnis umzudrehen nicht vorhanden, lautete eine sehr nüchterne Bestandsaufnahme. Die mögliche Reintegration Frankreichs in den militärischen Teil der NATO und die weitere Beziehung zwischen der ESVP und der NATO sowie das Zypern-Türkei Problem wurden schließlich als naheliegende Herausforderungen für die Europäische Union skizziert.

Insgesamt machte die Tagung deutlich, dass das zunehmende Ineinandergreifen von Fragen innerer und äußerer Sicherheit und die schwindende Trennschärfe zwischen den Politikfeldern eine langfristige Betrachtung dieser Schlüsselbereiche der Europäischen Union verlangt.

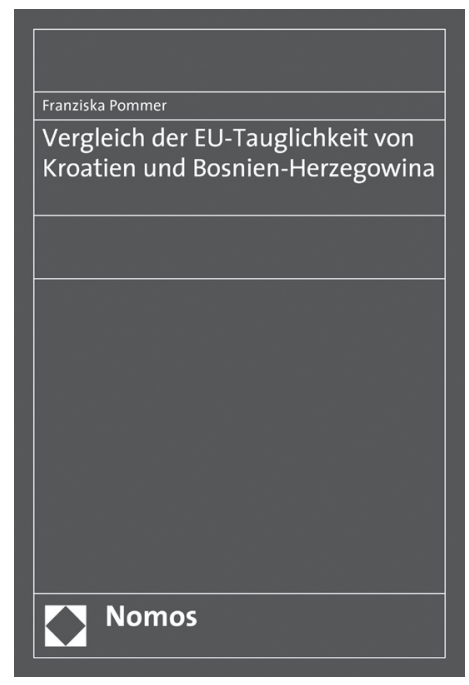

Bitte bestellen Sie bei Ihrer Buchhandlung oder bei Nomos Telefon 07221/2104-37| Fax -43| www.nomos.de | sabine.horn@nomos.de

\section{Vergleich der EU-Tauglichkeit von Kroatien und Bosnien- Herzegowina \\ Von Dr. Franziska Pommer, M.E.S. \\ 2008, 226 S., brosch., 44, $-€$, ISBN 978-3-8329-3644-O}

Der südosteuropäische Raum ist eine der größten Herausforderungen und Chancen für die europäische Staatengemeinschaft. Die Studie zeigt, wo derzeit die Probleme in Kroatien und Bosnien-Herzegowina liegen und welche Lösungen zum Erfolg führen können. Zudem werden die Anforderungen, die die EU an ihre zukünftigen Mitglieder stellt, analysiert und hinterfragt.



